\title{
DNA nanostructures fingerprint cellular organelles
}

\author{
DNA nanodevices that can enter cells and quantitatively image the levels of chemicals in organelles might find \\ applications in medical diagnostics.
}

\begin{abstract}
A $t$ the end of 2017, the field of DNA nanotechnology witnessed a leap forward when three papers were published showing the possibility of scaling up DNA origami structures at a cheaper cost than ever before ${ }^{1-3}$. In the short editorial that we published on that occasion $^{4}$, we remarked how, although this certainly represented the overcoming of a major roadblock in the field, not every application requires micrometre-sized DNA structures. For biological applications, smaller and simpler structures are often preferable. Their small size aids cellular uptake, their flexibility offers the chance of exploring various conformations and stimuli-responsive conformational changes, and their modularity offers the possibility of adding different functionalities on the DNA scaffold. Elegantly engineered DNA nanodevices have been proposed as probes in molecular diagnostics, cell sensing, drug delivery and imaging applications ${ }^{5}$.

For example, small DNA duplexes have been designed to report on the concentrations of secondary metabolites
\end{abstract}

in living cells and living organisms. These probes generally comprise fluorescence moieties responsive to particular chemicals, an internal standard for ratiometric quantification of the chemicals and possibly a targeting label to direct the DNA structures to specific organelles within the cells.

ChloropHore, the probe described in the paper published in the current issue of Nature Nanotechnology, measures the $\mathrm{pH}$ and the $\mathrm{Cl}^{-}$content within single lysosomes simultaneously and independently. Plotting these concentration values on a twodimensional map, the researchers obtained a chemical fingerprint of the lysosomes within cells, which led them to discover different organelle chemotypes. In particular, they identified a subclass of lysosomes that disappears in individuals suffering from the Niemann-Pick A, B or C diseases - a group of lysosomal storage disorders - and re-appears upon treatment of the cells with a known drug for these diseases.

From the same laboratory come the evocatively named DNA probes CalipHluor ${ }^{6}$ and cHOClate ${ }^{7}$, which measure $\mathrm{Ca}^{2+} / \mathrm{pH}$ and
$\mathrm{HOCl} / \mathrm{pH}$ in lysosomes and phagosomes, respectively. This series of sensors offers the opportunity of drawing a precise picture of the chemical status of organelles in specific circumstances, with ramifications for the fundamental understanding of cell biology and for possible medical applications. ChloropHore shows the possibility of using these devices to link the chemical identity of patients' cells and organelles to their health, and to monitor the effect of specific drugs and therapies. In other words, it introduces DNA nanotechnology into the world of personalized medicine.

Published online: 5 February 2019 https://doi.org/10.1038/s41565-019-0384-3

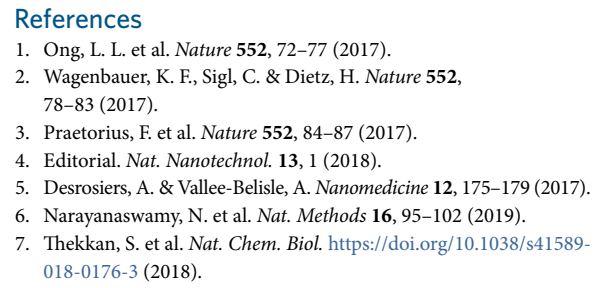

\section{Graphene macrostructures and the environment}

\author{
The connection between properties and environmental performance of graphene-derived structures is necessary to \\ develop materials that can improve industry standards.
}

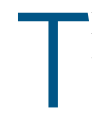
he main goal of nanotechnology is to study and apply the properties of structures measuring less than a few tens of nanometres across. In the collective imaginary, this often translates into the study of single nanostructures or a small number of them. If we take graphene, for example, a typical nanotechnology study concerns the development of electronic components based on one or more sheets of this carbon material.

For materials scientists and engineers, however, nanotechnology consists more in using the properties of individual nanostructures to develop new materials with improved performance. Going back to the example of graphene, or even better its derivative graphene oxide, realizing macroscopic porous structures is particularly promising for applications that require materials with large surface area combined with the facility of chemical modification, for example energy storage or water and air filtering. In a Review published in this issue, Nathalie Tufenkji and co-authors overview the efforts made in the past few years to demonstrate the potential of large structures composed of graphene and graphene oxide sheets for environmental applications.

The article covers applications of macroscopic graphene and graphene oxide structures for removal of contaminants from water, for desalination, for carbon dioxide capture, for air pollution treatment and for antimicrobial applications. Beyond overviewing the potential of graphene materials for these applications as demonstrated by proof of principle experiments, the real value of such an article lies in two other aspects. First, it explores the connection between the properties and the performance of the materials. Understanding this relationship is essential for the successful development of nanotechnology more generally, but too often it is neglected. Second, it points out the information that is currently lacking and that will be essential if these structures are intended to compete with current industry standards such as activated carbon. For example, for water cleaning applications, it will be necessary to explore the ability of graphene-derived macrostructures to remove organic pollutants or other emerging contaminants such as those connected to pharmaceutical compounds. Most importantly, the article stresses the need to move from relatively simple laboratory-scale studies to more complex and more realistic conditions, which is becoming a generally recognized necessity for environmental nanotechnology studies.

Published online: 5 February 2019 https://doi.org/10.1038/s41565-019-0385-2 